\title{
2 The Ganymede laser altimeter (GALA) key objectives, instrument 3 design, and performance
}

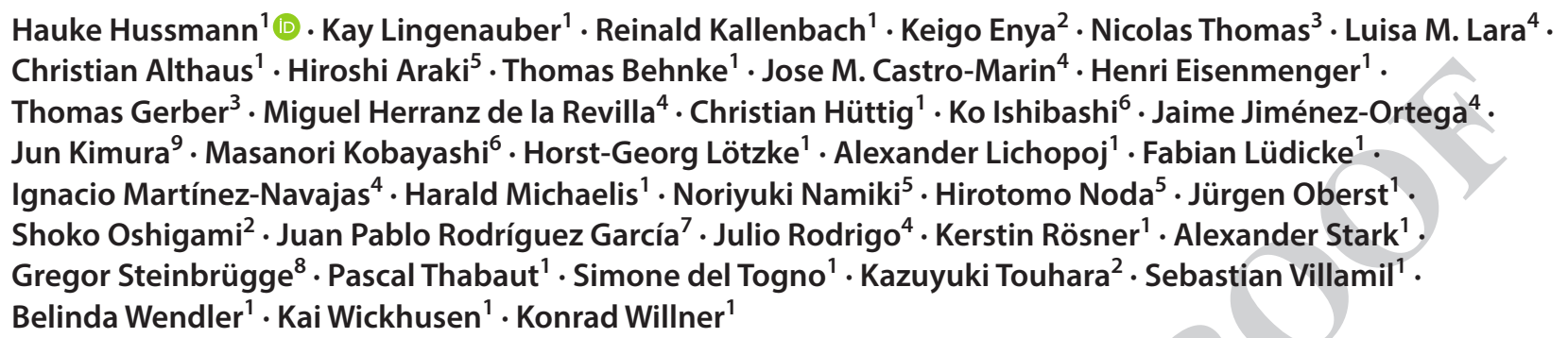

\begin{abstract}
The Ganymede Laser Altimeter (GALA) is one of the ten scientific instruments selected for the Jupiter Icy Moons Explorer (JUICE) mission currently implemented under responsibility of the European Space Agency (ESA). JUICE is scheduled for launch in mid 2022; arrival at Jupiter will be by end of 2029 with the nominal science mission-including close flybys at Ganymede, Europa, and Callisto and a Ganymede orbit phase - ending by mid 2033. GALA's main objective is to obtain topographic data of the icy satellites of Jupiter: Europa, Ganymede, and Callisto. By measuring the diurnal tidal deformation of Ganymede, which crucially depends on the decoupling of the surface ice layer from the deep interior by a liquid water ocean, GALA will obtain evidence for (or against) a subsurface ocean in a $500 \mathrm{~km}$ orbit around the satellite and will provide constraints on Ganymede's ice shell thickness. In combination with other instruments, it will characterize the morphology of surface units on Ganymede, Europa, and Callisto providing not only topography but also surface roughness and albedo (at $1064 \mathrm{~nm}$ ) measurements. GALA is a single-beam laser altimeter operating with up to $50 \mathrm{~Hz}$ (nominal $30 \mathrm{~Hz}$ ) shot frequency at a wavelength of $1064 \mathrm{~nm}$ and pulse lengths of $5.5 \pm 2.5 \mathrm{~ns}$ using a Nd:YAG laser. The return pulse is detected by an Avalanche Photo Diode (APD) with $100 \mathrm{MHz}$ bandwidth and is digitized at a sampling rate of $200 \mathrm{MHz}$ providing range measurements with a subsample resolution of $0.1 \mathrm{~m}$ and surface roughness measurements from pulse-shape analysis on the scale of the footprint size of about $50 \mathrm{~m}$ at $500 \mathrm{~km}$ altitude. The instrument is developed in collaboration of institutes and industry from Germany, Japan, Switzerland, and Spain.
\end{abstract}

Keywords Laser Altimetry · Solar System · Satellites of Jupiter · Ganymede · JUICE mission · GALA

\section{Abbreviations}

ADC Analog-to-digital converter

AEM Analog electronics module

APD Avalanche photo diode
BELA BepiColombo laser altimeter

BEO Back-end optics

CDR Critical design review

DC Direct current
Hauke Hussmann

hauke.hussmann@dlr.de

1 DLR Institute of Planetary Research, Rutherfordstr. 2, 12489 Berlin, Germany

2 ISAS/JAXA, Sagamihara, Japan

3 University of Bern, Bern, Switzerland

4 IAA-CSIC, Granada, Spain
5 National Astronomical Observatory Japan (NAOJ), Tokyo, Japan

6 Chiba Institute of Technology, Narashino, Japan

ESA, ESTEC, Noordwijk, The Netherlands

8 University of Texas, Austin, USA

9 Osaka University, Suita, Japan

\begin{tabular}{|l|l|l|l|l|}
\hline Journal : Large 12567 & Article No : $\mathbf{2 8 2}$ & Pages : $\mathbf{1 0}$ & MS Code $: \mathbf{2 8 2}$ & Dispatch : 10-10-2019 \\
\hline
\end{tabular}




$\begin{array}{lll}39 & \text { DPM } & \text { Digital processing module } \\ 40 & \text { ELU } & \text { Electronics unit } \\ 41 & \text { EM } & \text { Engineering model } \\ 42 & \text { EMC } & \text { Electro-magnetic compatibility } \\ 43 & \text { EMI } & \text { Electro-magnetic interference } \\ 44 & \text { EQM } & \text { Engineering qualification model } \\ 45 & \text { ESA } & \text { European space agency } \\ 46 & \text { FPA } & \text { Focal-plane assembly } \\ 47 & \text { FPGA } & \text { Field programmable gate array } \\ 48 & \text { GALA } & \text { Ganymede laser altimeter } \\ 49 & \text { JUICE } & \text { Jupiter icy moons explorer } \\ 50 & \text { LEU } & \text { Laser electronics unit } \\ 51 & \text { LHM } & \text { Laser head module } \\ 52 & \text { MLI } & \text { Multilayer insulation } \\ 53 & \text { M1 } & \text { Main mirror } \\ 54 & \text { M2 } & \text { Secondary mirror } \\ 55 & \text { MOS } & \text { Metal-oxide semiconductor } \\ 56 & \text { Nd:YAG } & \text { neodymium-doped yttrium aluminum garnet, } \\ 57 & & \text { Nd:Y }{ }_{3} \text { I }_{5} \mathrm{O}_{12} \\ 58 & \text { PCM } & \text { Power converter module } \\ 59 & \text { PFM } & \text { Proto flight model } \\ 60 & \text { RFM } & \text { Range finder module } \\ 61 & \text { RX } & \text { Receiver } \\ 62 & \text { RXM } & \text { Receiver module } \\ 63 & \text { STM } & \text { Structural and thermal model } \\ 64 & \text { TEL } & \text { Receiver telescope } \\ 65 & \text { TID } & \text { Total ionizing dose } \\ 66 & \text { TRU } & \text { Transceiver unit } \\ 67 & \text { TX } & \text { Transmitter } \\ & & \end{array}$

\section{Introduction}

The Galileo spacecraft was the first to enter Jupiter orbit (1995-2003) for an in-depth exploration of the planet and its satellite system [4]. From the data of this historic mission, one of the most important conclusions was that the icy moons of Jupiter-Europā, Ganymede, and Callistocontain global subsurface water oceans underneath their icy crusts (e.g., $[14,24])$. Whereas subsurface oceans fit well into the concept of the differentiated satellites Europa and Ganymede, it is not quite understood how Callisto could maintain an ocean albeit being only partially differentiated. However, based on measurements of the induced magnetic fields that are generated in response to the external Jovian magnetosphere, all three icy satellites are believed to contain global subsurface water oceans $[10,12]$. The possibility is intriguing that these large liquid water oceans represent habitable environments. Investigation of these environments is one of the main motivations for ESA's Jupiter Icy Moons Explorer (JUICE) mission [8].

The Ganymede Laser Altimeter (GALA) as part of the JUICE payload is one of the instruments focusing on the presence and characterization of the subsurface water ocean on Ganymede. Obtaining topographic data of Europa, Ganymede, and Callisto are the main purpose of GALA. Whereas measurements at Europa and Callisto will be restricted to several close flybys, the measurements at Ganymede will be acquired in an orbital phase at an altitude of $500 \mathrm{~km}$ from the surface of the satellite. Measuring the tidal deformation at Ganymede on its diurnal cycle of 7.15 days is another key objective for GALA. The latter will be indicative of whether Ganymede possesses a subsurface ocean beneath its thick (about $100 \mathrm{~km}$ ) ice shell [14, 24]. Furthermore, the measurements can provide constraints on the thickness of Ganymede's outer ice shell $[11,26]$.

GALA is a single-beam altimeter: a laser pulse (at 1064 $\mathrm{nm}$ wavelength) is emitted using an actively Q-switched $\mathrm{Nd}$ :YAG laser firing at $30 \mathrm{~Hz}$ in nominal operation. A small fraction of the pulse is guided through fiber optics onto the detector characterizing the outgoing pulse and time of emission. Typically after a few msec, the Lambertian reflection of the pulse from the surface of Ganymede is received by a 25-cm aperture telescope and transferred to the detector, an Avalanche Photo Diode (APD). The signal is digitized at a sampling rate of $200 \mathrm{MHz}$ and transferred to the range finder module, which determines (a) the time of flight between emitted and received pulse (b) the pulse shape, in particular the pulse width and (c) the energy of the received pulse. From the time of flight of the wave package (converted into range) and the spacecraft position and attitude, the height above a reference surface in post-processing of the data can be determined with an accuracy of a few meters [26]). By collecting several hundred millions of shots during the nominal mission, the topography of Ganymede's surface will be obtained from the range measurements. The periodic time-variability of surface heights at different anomalies of Ganymede's orbit around Jupiter will reveal the tidal deformation and physical librations of the satellite's rotational state. With maximum tidal double amplitudes of about 7-8 $m$ in case of an ocean on Ganymede and only a few tens of $\mathrm{cm}$ in case of an ice shell that is directly connected to a deeper silicate layer [14], the tidal signal is indicative of a subsurface ocean.

The pulse width is a measure of the surface roughness and slope at the scale of the laser footprint (50-m diameter at 500-km altitude orbit). From the shape of the transmitted and returned pulse, the broadening of the pulse can be determined which will then provide a measure for the small-scale roughness combined with the general slope. By subtracting the general slope that can be measured by the sequence of laser spots at a given track for specific baselines, the smallscale roughness within the laser footprint can be extracted. To compare surface roughness maps with boundaries of geologic units provides insight into processes that have modified the terrain on different scales. Whereas surface roughness 
maps have been obtained from altimetry measurements for the terrestrial planets and the moon (e.g., [23, 28], these data sets are not yet available for the icy satellites. An estimate of the albedo at the laser wavelength is obtained by comparing the energy of the emitted pulse with the one of the received pulse.

The JUICE mission: JUICE is scheduled for launch from Kourou, French Guyana, in 2022, and it will be arriving at the Jupiter system by late 2029. Details on the trajectory and mission analysis can be found in [5]. After Jupiter orbit insertion using a Ganymede flyby, the spacecraft will go into Jupiter orbit for about 3 years completing several close flybys at Ganymede, Europa, and Callisto. These flybys are crucial for investigating the satellites, but are also used for navigation purposes in the Jovian system. By the end of 2032, JUICE will enter Ganymede orbit starting with an elliptical $200 \times 10,000 \mathrm{~km}$ near-polar orbit. Due to perturbations by Jupiter, the orbit will naturally evolve into a 5000 $\mathrm{km}$ circular orbit. After that, it will again evolve into the elliptical orbit. The elliptical phase (including the 5000-km circular phase) will last for about 150 days. With a maneuver at pericenter, the spacecraft will then enter the final 500$\mathrm{km}$ circular low-altitude orbit. This final phase will last for about 132 days - mainly constrained by the radiation dose received by the spacecraft and instruments - and is - apart from the close flybys - the most important phase for GALA measurements. The design and technical parameters of the instrument are optimized for the latter $500-\mathrm{km}$ orbit around Ganymede. If resources allow for a continuation of the mission, a 200-km orbit would be a possible mission extension.

In the following, we will briefly summarize scientific key objectives of GALA (Sect. 2) and describe the instrument design in detail in Sect. 3. The instrument performance is summarized in Sect. 4 followed by the final Conclusions section.

\section{Scientific objectives}

A fundamental goal of exploratory planetary missions like JUICE is to characterize and measure the figure, topography, and rotation of the target bodies. This is essential for understanding both the interior state and global aspects of satellite evolution as well as regional and local processes that have shaped the body's surface. A state of the art tool for this task is a laser altimeter (e.g., [20, 21, 23, 34]), because it can provide absolute topographic height and position with respect to a Ganymede (or Europa/Callisto) centered coordinate system. From gravity field determination by the Galileo mission, the moment of inertia of Ganymede has been determined $[2,18]$. Its dimensionless value of 0.3115 \pm 0.0028 shows a considerable degree of differentiation and corresponding interior structure models consist of an iron core, a silicate shell, a high-pressure ice shell, a subsurface ocean, and an outer ice-I layer (e.g., [2, 18, 25]). The existence of a metallic core is further supported by the presence of a magnetic dipole field detected by Galileo [12]. Based on these interior structure models, the tidal response of Ganymede on its eccentric orbit around Jupiter can be calculated assuming rheologic parameters of the planetary material, metal, rock, and ices $[9,14]$. Based on these models, maximum tidal double amplitudes at Ganymede's surface of more than $7 \mathrm{~m}$ are expected in case of a decoupling of the outer ice shell by a liquid water ocean from the deep interior. In case of a completely solid ice shell, tidal amplitudes would barely reach a few tens of $\mathrm{cm}$. The radial deformation by these eccentricity tides is usually parameterized by the second-order tidal Love number $h_{2}$. Based on current performance models of GALA, Ganymede's $h_{2}$ Love number will be determined with a relative accuracy of about $2 \%$. This would be sufficient to determine whether the ocean is there and it would further constrain the ice thickness by $\pm 20 \mathrm{~km}$ [26]. The total ice thickness of Ganymede's outer ice shell is uncertain. Estimates are on the order of about $100 \mathrm{~km}$ (e.g., $[14,24]$ ). In addition to $h_{2}$, changes in Ganymede's rotation rate - the longitudinal librations - are indicative of a subsurface ocean. The libration amplitudes can be detected with GALA with an accuracy of 2.5-6.6 $\mu \mathrm{rad}$ corresponding to constraints on the ice thickness of about 24-65 km [27].

The geology of Ganymede is unique among the icy satellites and very diverse [17]. The surface has been highly modified by tectonic processes and extensional features. In addition, there are impact craters of all types and sizes, including also relaxed craters that show evidence of strong thermal activity in the satellites' past [19]. Two terrain types - termed bright and dark terrain, respectively - are regionally distinct on the surface. The heavily cratered ancient dark terrain covers about one-third of the surface, whereas the more recent and heavily tectonized bright terrain covers two-thirds [17]. Topographic data of Ganymede's surface are currently available only from Galileo imaging data based on stereo photogrammetry (e.g., [7]) and photo-clinometry [19]. These data sets are extremely limited in terms of coverage and the large-scale topography on Ganymede is completely unknown. With a radial accuracy of a few meters GALA will obtain highly accurate topographic data that will advance our understanding of the processes that have shaped the satellite's surface. In addition, information on the physical state of the shallow subsurface at time of formation of these features will be gained from topographic data.

With respect to evolution of the Galilean Moons, GALA aims at

- an exploration of the morphology and surface geology of Ganymede, Europa, and Callisto, 
- a determination of their interior structures from a combination of shape, topography, and gravitational field data from the radio science experiment $3 \mathrm{GM}$,

- the exploration of their surface chemistry and physical properties,

- and at their formation and evolution especially with respect to subsurface water oceans (see also [11, 26]).

To this end, precise geodetic measurements to determine the shapes of the moons and their topography are mandatory. The topography is needed:

- to account for the effects of topographic heights on the gravity field and to account for near surface mass distribution anomalies above the reference figure [16, 30],

- to support geological studies, e.g., to identify and characterize tectonic and cryovolcanic regions on the icy moons,

- to measure the radial tidal deformation on the 7.15-day diurnal cycle to detect and characterize a potential subsurface ocean on Ganymede.

Investigations by GALA will furthermore contribute to determine:

- the orientation and rotational states of Ganymede, Europa, and Callisto, in particular the physical librations of Ganymede's outer ice shell in combination with imaging data (JANUS) and Radio Science data (3GM);

- surface characteristics (roughness, slopes, and albedo) on Ganymede, Europa, and Callisto.

GALA will form an integral part of a larger geodesy and geophysics package, incorporating radio science, imaging systems, and subsurface radar. The synergy between these and the other payloads of the JUICE mission will provide an in-depth study of Gaynmede and an assessment of its potential habitability in concordance with the overall JUICE science objectives [8].

\section{Instrument design}

Regarding solar system exploration, several laser altimeters have been flown investigating Mars [20, 32], the Moon [3, $13,21,31]$, Mercury [22, 34], and various asteroids [6, 15, 33]. The instrument designs are heavily dependent on the specific purpose of the mission and on the environmental conditions during cruise and at the target. In this section, the GALA design at the time of the Instrument Critical Design Review that was successfully completed in April 2019 is described.

\subsection{Design overview}

The GALA development is based on heritage from the BepiColombo Laser Altimeter (BELA), which was built by a similar consortium. Descriptions of BELA are given in [29] and by Thomas et al. (2019, this issue). Compared to BELA, several aspects had to be optimized due to the differences in environments and scientific objectives at Jupiter as compared to Mercury. Whereas the overall technical realization is very similar, using similar electronic units and the same digital range and pulse-shape detection schemes, main differences are the following: GALA uses a higher shot rate (30 $\mathrm{Hz}$ nominal, $50 \mathrm{~Hz}$ maximum) as compared to the $10-\mathrm{Hz}$ frequency of BELA, which was required for keeping the interpolation error at cross-over points (crossing laser tracks at Ganymede's surface) sufficiently small for the error budget of the tidal measurements [26] and for obtaining sufficient along-track coverage at close - and thus fast-flybys. The bandwidth of the GALA detector is $100 \mathrm{MHz}$ as compared to $20 \mathrm{MHz}$ for BELA. This together with an enhanced sampling rate of $200 \mathrm{MHz}$ for the pulse digitization will allow for improved characterization of the return pulse shape, which will provide invaluable information on roughness and reflectivity of Ganymede's icy surface. With an altitude of $500 \mathrm{~km}$ the circular Ganymede orbit is closer than the 480 $\times 1500 \mathrm{~km}$ elliptical Mercury orbit of BepiColombo which reduces the required laser energy that is transmitted. GALA has an output of $17 \mathrm{~mJ}$ compared to $50 \mathrm{~mJ}$ of BELA.

The different thermal and radiation environments had the following impact: The GALA design is more compact to take advantage of shielding by mechanical parts in addition to spot shielding that was necessary for exposed components of the transceiver unit. Specific materials have to be used (e.g., for the optical components to avoid darkening due to radiation effects). For JUICE, the electronic boxes are located inside a vault together with electronic boxes from other instruments and are, therefore, located quite remotely from the optical unit. A particular challenge is the qualification of optical components of the transceiver system. Therefore, extensive electron irradiation testing has been performed with the optical and electro-optical components of the GALA transceiver system. In addition, the baffle systems had to be designed differently due to the differing thermal and stray-light conditions at Mercury and Jupiter.

At the time of this writing, GALA had successfully passed the Critical Design Review (CDR). It is essential that the design is verified by tests that guarantee full functionality and performance of the instrument. An engineering model (EM) has been delivered which is representative with respect to the electrical interfaces to the spacecraft. The EM can be fully commanded (including laser firing) and is used for full-functional tests. In addition, the structural thermal model (STM) is built, which undergoes 
mechanical testing and thermal cycling. Currently, the (proto-)flight model (PFM) is under development. For several subunits, engineering qualification models (EQMs) are built which will undergo substantial testing for verifying the functionality and robustness of these units. The planned verification and calibration campaigns are based on procedures used for BELA. Detailed descriptions can be found in [1] and in Thomas et al. (2019, this issue). The GALA instrument consists of three units which incorporate several subassemblies (see Fig. 1):
1. The Transceiver Unit (TRU) (see Fig. 2) houses the laser optics (Laser Head Module, LHM) and the appropriate laser head electronics. The laser emits the laser pulse through the collimating transmitter telescope. The return laser pulse is received by the receiver telescope (TEL) and focused on the detector (Si-APD) by the back-end optics (BEO). The Analog Electronics Module (AEM) pre-amplifies and digitizes the detector output before it is directed to the Range Finder Module (RFM) inside the Electronic Unit (ELU). The TRU includes a baffle that protects the receiver telescope against straylight from
Fig. 1 Block diagram of the GALA instrument showing key elements of the design. The acronyms Tx and Rx stand for transmitter and receiver, respectively
Fig. 2 Overview of the GALA instrument. GALA contains three main units: the transceiver unit (TRU), the Electronics Unit (ELU), and the Laser Electronics Unit (LEU). Responsibilities in the consortium consisting of institutes and industry from Germany, Japan, Switzerland, and Spain are indicated in the upper left part
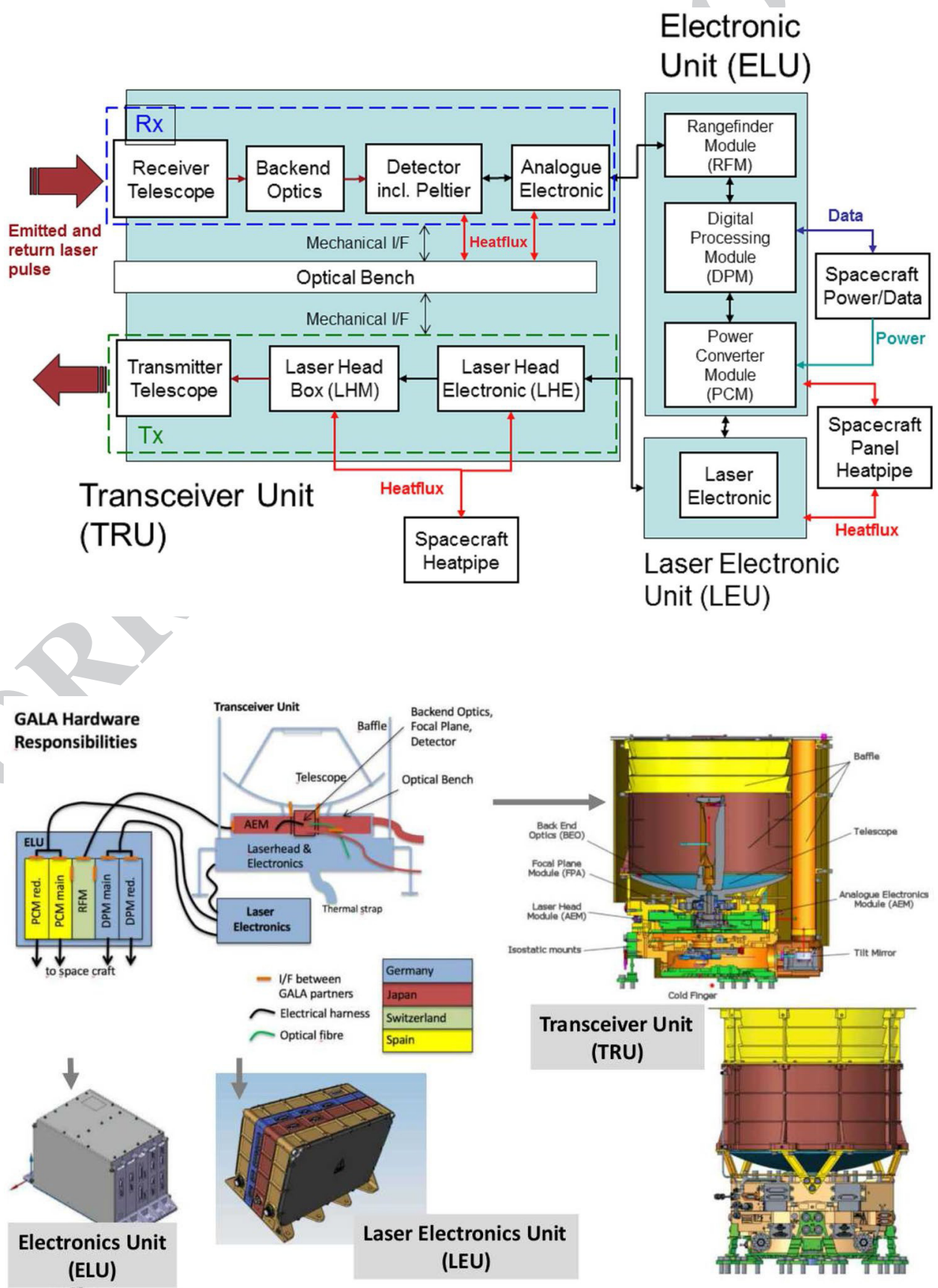

\begin{tabular}{|l|l|l|l|l|}
\hline Journal : Large 12567 & Article No : 282 & Pages : 10 & MS Code : 282 & Dispatch : 10-10-2019 \\
\hline
\end{tabular}


Jupiter's atmosphere, from surface reflections from the target outside the laser footprint, and from reflection of light by other parts of the spacecraft. The baffle furthermore prevents laser straylight from direct incidence to the receiver telescope and from hitting other parts of the spacecraft.

2. The Electronic Unit (ELU) houses the digital rangefinder module (RFM), which analyzes the detector signal and computes range, and pulse shape for the analysis of surface slope and roughness. The Digital Processing Module (DPM) is the main computer of the instrument and controls all instrument functions as well as the interface to the spacecraft. The Power Converter Module (PCM) provides power in different voltages for all instrument assemblies.

3. The Laser Electronic Unit (LEU) consists of the laser control module, the laser diode driver, and the power distribution module. These include capacitors, highvoltage supply, and power driver for the laser pump diodes. Electrical interfaces to the spacecraft for power and data are via the Electronic Unit. Thermal interfaces to the spacecraft are via heat pipes, surface contacts to the spacecraft panel (conductive), and the three units outer surfaces to the spacecraft (radiative).

\subsection{The transceiver unit}

Figure 2 shows the design concept of the transceiver unit which includes the laser transmitter (TX) and the receiver (RX) with their respective protection baffles. The GALA TRU comprises:

- the Receiver Module (RXM) including the Cassegrain receiver telescope (TEL) with Optical Bench, the provided Back-End Optics/Focal-Plane Assembly (BEO/ FPA), and Analog Electronics Module (AEM). Optical bench and AEM housing form one integral structural part;

- the Laser Head Module (LHM) including two laser transmitters in cold redundancy configuration, the beam expander, laser head electronics with Pockels cell drivers, energy monitors, temperature sensors, and the quasi-isostatic mounts for connection respectively as mechanical interface to the optical bench of the spacecraft,

- the thermal interface of the LHM to the spacecraft mounting-plate;

- all electrical harnesses connecting the LHM to the LEU which powers and controls the laser including its optical and thermal performances; the LEU resides in the spacecraft vault, which protects the electronic boxes of several instruments from radiation;

- the fiber-optical harness for the start pulse detection to the RX detector,
- the thermal hardware including the protection baffles, multilayer insulation (MLI) and survival, decontamination, and switch-on heaters with temperature sensors.

LHM, RXM, and their subassemblies will be integrated onto the TRU under the requirements on co-alignment and instrument internal electro-magnetic compatibility. The LHM and the LEU can be operated and tested as a laser system independent from the other subunits.

Proper operation of the TRU is guaranteed within the specified operational temperature ranges between +5 and $+30{ }^{\circ} \mathrm{C}$ provided by the spacecraft thermal control system without any additional instrument thermal control hardware such as an operational heater. Only the Si APD sensor has its dedicated Thermo-Electric Cooler to control its temperature precisely to $25^{\circ} \mathrm{C}$.

\subsubsection{Design of the receiver module}

The receiver module (RXM) is one of the submodules of the TRU. It consists of the receiver telescope (TEL), BackEnd Optics (BEO) with relay optics and field stop aperture, Focal-Plane Array (FPA) with Si-APD and detector related electronics, the optical fibers to the FPA for the start pulse detection and the Analog Electronics Module (AEM), consisting of FPA control, analog signal processing, analog-todigital converter, and digital signal processing.

The optical design of the TEL comprises two mirrors (M1 and secondary mirror M2), which form a Ritchey-Chretien telescope with a focal length of $1200 \mathrm{~mm}$. The back focal distance is positioned at $10 \mathrm{~mm}$ of the vertex to the intermediate image plane behind the M1 apex. The free entrance pupil diameter, respectively, the effective receiver diameter of at least $250 \mathrm{~mm}$, is equivalent to the un-obstructed area of the mirror M1. The transmission of the receiver telescope into the field stop aperture is influenced by the reflectivity of the mirrors at $1064 \mathrm{~nm}$, various alignment errors in addition to image blur. The total reflectivity of the telescope mirrors will be at least 95\% (begin of life). The resulting optical transmission including the effects of these deviations but without degradation effects caused by contamination and/or particles will be at least $86 \%$. The end-of-life optical transmission of the telescope is budgeted with $83 \%$. The receiver field of view (full cone) is fixed by the diameter of the field stop aperture and the focal length of the telescope. The value is limited to less than $580 \mu \mathrm{rad}$ including all manufacturing and assembly tolerances as well as environmental loads. The transmitter has a beam divergence of $100 \mu \mathrm{rad}$. The pointing requirement is $145 \mu \mathrm{rad}$, which is about $50 \%$ larger than the beam divergence. The receiver has to capture the return pulse, i.e., its field of view must be larger than the beam divergence and the alignment between transmitter
427 
and receiver must be stable. The value of $580 \mu \mathrm{rad}$ for the receiver field of view accounts for the beam divergence and includes margins for cross-alignment and thermo-elastic distortions.

All materials for the telescope are selected to provide sufficient radiation hardness against high-energy radiation (electrons, protons, and gamma rays) to the levels experienced at the TEL during GALA mission. The material of the mirrors M1 and M2 together with the reflective coating in combination with the strut material is chosen for optimum adaption and athermalization to ensure the optical performance over the operational temperature range.

The Si APD Detector Key parameters of the Si Avalanche Photo Diode (APD) are the responsivity of $750 \mathrm{kV} / \mathrm{W}$ in a bandwidth of about $100 \mathrm{MHz}$, the noise spectral density of typically $20 \mathrm{fW} / \sqrt{\mathrm{Hz}}$ varying from module to module, and the rise time and fall time of $3.5 \mathrm{~ns}$ to meet performance requirements on the receiver. For flight, an APD module with noise spectral density of less than $40 \mathrm{fW} / \sqrt{\mathrm{Hz}}$ will be selected. The latter value is the upper limit for full science performance of the GALA instrument which must not be exceeded.

The APD itself has undergone extensive electron irradiation testing. The main problem is the susceptibility to electron ionizing dose. Electrons presumably cause higher radiation damage at the $\mathrm{Si} / \mathrm{SiO}_{2}$ interface layer than protons. This phenomenon is well known from MOS technology and very much depending on the thickness of the oxide layer. The main effect of the electron total ionizing dose (TID) is an enhanced surface dark current which compromises the APD performance much less than an increased bulk dark current. Surface dark current extends in frequency from typically a few $\mathrm{Hz}$ up to about $1 \mathrm{kHz}$ (1/f noise typical at an interface capacitance). Test results indicate that the bulk dark current is orders of magnitude lower than the surface dark current. Overall, the Si APD still meets the spectral noise density requirement of $40 \mathrm{fW} / \sqrt{\mathrm{Hz}}$ in the frequency range $100 \mathrm{kHz}$ to $100 \mathrm{MHz}$ for full scientific performance after representative electron irradiation.

The APD detector produces a single-ended electronic timing signal with an analog bandwidth of $100 \mathrm{MHz}$ and a noise floor of about $20 \mathrm{nV} / \sqrt{\mathrm{Hz}}$. The timing pulses have temporal widths ranging from a few ns to about 100 ns driven by surface topography roughness and slope of the terrain on Ganymede. The signal-to-noise ratio (SNR) depends on the distance of the spacecraft to the planetary surface and the surface albedo and slope. Assuming a flat surface with an albedo of 0.45 (average on Ganymede), the SNR can be as high as 100 at close encounters of 100-km altitude (during flybys), but also as low as 2.5 at an altitude of $1400 \mathrm{~km}$.

To adapt the noise floor and signal strength to the dynamic range of the analog-to-digital converters (ADCs), the gain can be switched in two steps. The single-ended signal originating from the APD assembly in the FPA sensor is converted to a differential signal and fed to differential ADCs. The data are transferred real time in 12-bit parallel streams to the RFM (Range Finder Module) where they are processed by a dedicated algorithm. The AEM follows the RFM as slave regarding the timing. The clock signal from the RFM is transferred to the AEM for timing the ADCs which both operate at 100 Msamples/s. This way, the detected start and return pulses from the APD detector are correctly marked in time to derive the ranging measurement from the time of flight. The start pulse from the laser is fed to the APD sensor through fibers and the return pulse from the planetary surface is fed to the APD detector through the TEL and the BEO. The amplifier for the signal originating from the APD detector module has a bandpass which is flat between $100 \mathrm{kHz}$ and $100 \mathrm{MHz}$. It may be decided in course of phase $\mathrm{C}$ and $\mathrm{D}$ to limit the bandwidth to eventually exclude electro-magnetic interference from power converters without limiting scientific performance. EMC testing with the GALA engineering model revealed that there is no such interference.

\subsection{The laser head module}

The laser consists of (1) a diode pumped cavity with a laser crystal (Nd:YAG) which is side pumped by two laser diode arrays, (2) an electro-optical Q-switch (Pockels Cell) which triggers the generation of the required short pulses, and (3) optics for an unstable laser resonator with a graded reflectivity out-coupling mirror enabling low transversal mode operation with near diffraction limited beam quality of $\mathrm{M}^{2}<2.3$.

The mechanical/thermal design of the laser resonator includes a three-stage decoupling configuration of the laser resonator from the LHM to get a stable running laser source under mechanical and thermal operating conditions. The actual laser resonator consists of three mirrors-end mirror, folding mirror, out-coupling mirror-and two wave plates which are directly mounted at the laser resonator bench. As far as possible, the laser resonator bench is thermally and mechanically decoupled from the rest of the LHM, i.e., there is no direct heat dissipation from either the laser diode arrays or the laser rod into the laser resonator bench. Mechanically, the laser resonator bench has four small bolt areas as an interface to the transmitter optical bench to get an optimum thermal isolation from each other. All other laser resonator optics (polarizer, laser rod, and Pockel's cell) except the laser diode arrays were mounted directly to the transmitter optical bench. The transmitter optical bench also provides a stable platform for the remaining beam guiding optics outside the laser resonator. Also the transmitter optical bench only has three mechanical interfaces to the base unit. Therefore, it is similarly decoupled from the base unit as the laser resonator bench from the optical bench.
535 
The pump laser diode arrays, which are the most intense heat sources inside the laser resonator, are directly mounted on the LHM. From the LHM, the heat is removed via the transmitter cover and the thermal strap to the spacecraft cold finger interface. The conduction cooled cavity design consists of the Nd:YAG rod, which is side pumped, the related housing, and mounting pieces. The predefined size of the LHM requires a small package of the laser rod unit optimized for high efficiency and a wide operational temperature range. Compared to the design of the BELA laser rod assembly, the number of laser diode arrays is reduced from four arrays to two arrays. This reduction was possible due to the availability of assemblies that can operate at $200 \mathrm{~A}$ of diode laser current, whereas the BELA diode lasers only were operated at $100 \mathrm{~A}$.

\subsection{The laser electronics unit}

The laser electronics unit (LEU) (see Fig. 2) contains all laser electronics and controls all laser operations:

- Laser diode drivers, main, and redundant.

- Laser control module.

- Power distribution module.

It has a data interface to the electronics unit (ELU), but no direct electrical interface to the spacecraft. The LEU generates high pump current (200 A) for the laser pump diodes inside the LHM. It contains capacitor reservoirs for providing the laser pump energy. Inside this electronics box, different subassemblies are mounted in frames, which are mated together to form the LEU. The LEU has to be accommodated in close vicinity to the TRU, since the harness length of the laser power cables should be kept as small as possible. With respect to BELA, some major improvements in the electronics design concept have been implemented. These include the separation of high-power electronics circuits from sensitive signal electronics into different compartments and into secondary circuit groups with separated grounding references. This reduces the cross-talk from the high-power electronics of the laser system to low-noise electro-optical sensor electronics needed for processing the APD signal or the energy monitoring of the laser pulse, or any precise control voltages such as temperature sensor read-outs. In addition, the phase margins of receiver amplifiers or the servo-loops for the voltage level controls have been analyzed and measured carefully in order to improve their robustness against electro-magnetic interference.

\subsection{The electronics unit}

The electronics unit (ELU) (see Fig. 2) is a box containing three modules, the Digital Processing Module, the
Rangefinder Module, and the Power Converter Module. It is located inside the vault of the spacecraft to protect the electronics from radiation damage.

The digital processing module (DPM) is the instrument processing and control front end. It is located together with the Power Converter Module (PCM) and the Range Finder Module (RFM) in the ELU. The DPM contains the on-board software which consists of two major parts, the Boot Software and the instrument Application Software. The DPM will perform the following tasks:

- Reception of telecommands and their decoding.

- Reception and processing of the digitized receiver signal Control of the sensor operation.

- Telemetry formatting and transmission.

- Housekeeping data acquisition.

- Instrument health check.

- Software upload support.

The range finder module ( $R F M)$ is the digital receiver part of the GALA instrument and it uses similar techniques as developed for the BepiColombo Laser Altimeter (BELA) ([29] and Thomas et al. in this issue). It is included in the Electronics Unit (ELU) which also contains the Digital Processing Module (DPM) and the Power Converter Module (PCM) (see Fig. 1. It receives the digitized APD sensor data from the two analog-to-digital converters (ADCs). The time series has 12 bit data every $5 \mathrm{~ns}$ due to parallel $100 \mathrm{Mbps}$ sampling with a phase shift of approximately $180^{\circ}$. The RFM controls these two ADCs inside the AEM in a master-slave configuration. It sends its $100 \mathrm{MHz}$ clock signal to the clock ports of the two ADCs. This clock signal is used to count the 5 ns time steps between the optical laser start pulse and the optical return pulse from the planetary surface both detected by the APD. This way, the time-of-flight of the optical pulse from the GALA instrument to the planetary surface and back to the GALA receiver is measured. This means that the RFM processes both the digitized laser transmitter (TX) and return pulse data (RX) sent from the AEM to the RFM. GALA uses an accurate Pulse Per Second (PPS) signal, which is retrieved directly from the spacecraft oscillator, to calibrate the range finder clock which is stable on short time-scale, but will drift on long time-scales. As the spacecraft oscillator is continuously monitored during ground contacts and stable on long time-scales, the validity of the GALA time-of-flight data can be ensured through the complete duration of the mission.

The power control module (PCM) is responsible for generating regulated supply voltages for all the GALA instrument subsystems from the spacecraft regulated bus voltage. It consists of two independent boards (main and redundant) in cold spare configuration. The PCM interfaces the spacecraft power subsystem, with two (one nominal and one 
redundant) regulated power lines on separate connectors. In case of failure, both the nominal and the redundant power lines can be applied simultaneously, so PCM will include isolation to avoid loss of one power source by a failure in the other power source. Input voltage from the spacecraft is $28 \mathrm{~V}$. The PCM generates and distributes the following secondary voltages: $+3.35 \mathrm{~V}, \pm 7.0 \mathrm{~V}$, and $+12 \mathrm{~V}$. The high efficiency (better, or equal, than 75\%) PCM is designed with several kinds of protections (input bus under-voltage; DC/ DC converter over-voltage, under-voltage, and over-load). Moreover, single-event protections for radiations impacts are also contemplated. As for the other modules and units of the GALA instrument, the effects of single-event latch-up, gate rupture, burn-out, upset, transients, and functional interrupt have been analyzed applying a radiation model, worst-case scenarios, and available data of the electronics components. Regarding destructive events, a linear energy transfer threshold of $60 \mathrm{MeV} /\left(\mathrm{mg} \mathrm{cm}^{2}\right)$ is required. For components with lower threshold, a detailed analysis on the probability for the different single events has been performed and the criticality has been judged. For FPGAs, the triple redundancy concept was used.

The FPGA included in this module is also capable to provoke a DC/DC converter re-start via an isolated digital interface among other functionalities. Finally, soft-start circuits and EMI filters are also built-in.

\section{Instrument performance}

The diversity of targets and the different phases of the trajectory including flybys and orbital phases during the course of the JUICE mission require flexibility of the instrument to achieve the various scientific objectives in different contexts. GALA will be capable of acquiring range measurements at distances of $1600 \mathrm{~km}$ at Europa, $1400 \mathrm{~km}$ at Ganymede, and $1100 \mathrm{~km}$ at Callisto, respectively. Different albedo values of the respective surfaces are determining the maximum distances (with all other parameters being equal). However, due to degradation in the course of the mission and due to surface roughness and slopes, some of the return signals might be lost at these maximum distances. Instrument parameters, in particular the laser energy and shot frequency but also the receiver telescope diameter, are optimized to give best performance at altitudes of about $500 \mathrm{~km}$ at Ganymede during the last phase of the mission, i.e., during the $500-\mathrm{km}$ orbit. Key characteristics of the instrument are shown in Table 1. The time-of-flight measurements will be accurate to about 0.67 ns corresponding to a range resolution of 0.1 $\mathrm{m}$ which is about one order of magnitude less than expected radial orbit errors. However, in addition to the instrument's resolution, there are other factors, e.g., pointing accuracy, and errors in the orbital position of the spacecraft at the
Table 1 Key characteristics of the GALA instrument

\begin{tabular}{ll}
\hline Parameter & Value \\
\hline Laser energy & $17 \mathrm{~mJ}$ \\
Wavelength & $1064 \mathrm{~nm}$ \\
Nominal shot frequency & $30 \mathrm{~Hz}$ \\
Maximum shot frequency & $50 \mathrm{~Hz}$ \\
Pulse width (1- $\sigma$ ) & $2.9 \mathrm{~ns}$ \\
Beam divergence (full cone & \\
At 1/e intensity) & $100 \mu \mathrm{rad}$ \\
Telescope radius & $12.5 \mathrm{~cm}$ \\
Field of view (full cone) & $580 \mu \mathrm{rad}$ \\
\hline
\end{tabular}

time of the measurement driving the accuracy to orders of a few meters. The instrument is designed for obtaining range measurements with an accuracy $<1 \mathrm{~m}$ at $500-\mathrm{km}$ altitude at an albedo of 0.2 and $20^{\circ}$ surface slope on Ganymede. The latter values have been used as a standard case, applicable for the orbit and main target of the JUICE mission (see also $[11,26])$.

To avoid gaps in along-track direction when taking highresolution height profiles during flybys or at specific targets in the GCO-500 phase, GALA will use a high shot frequency of up to $50 \mathrm{~Hz}$. The nominal shot frequency for which the laser energy is optimized is $30 \mathrm{~Hz}$ and will be mainly used during the 500-km orbit. Each shot will provide a measure of the surface roughness at footprint scale of about $50 \mathrm{~m}$ at 500-km altitude. For that purpose, GALA will be capable of digitizing and analyzing the return pulse which will be sampled at a frequency of $200 \mathrm{MHz}$.

\section{Conclusions}

By characterizing in detail Jupiter's moon Ganymede, the JUICE spacecraft will be the first mission to orbit a satellite of the Solar System other than the Earth's Moon. As part of the mission several flybys at Europa and Callisto will deepen our understanding of the current state and evolution of the Jovian satellite system. GALA which is developed in collaboration of institutes and industry from Germany, Japan, Switzerland, and Spain will provide fundamental knowledge about Ganymede, Europa, and Callisto and will, in combination with other instruments, lay the ground for further exploration of the Galilean Satellites by future in-situ missions (e.g., landers or penetrators).

Acknowledgements The authors would like to thank two anonymous reviewers for their helpful comments on a first version of the manuscript. Financial support was provided under grant 50 QJ 1401 on behalf of the DLR Space Administration by the German Bundesministerium für Wirtschaft und Energie. This research has been supported by

\begin{tabular}{|l|l|l|l|l|}
\hline Journal : Large 12567 & Article No : 282 & Pages : 10 & MS Code : 282 & Dispatch : 10-10-2019 \\
\hline
\end{tabular}


the Spanish Ministerio de Economía y Competitividad under Contract ESP 2016-76076-R.

\section{References}

1. Althaus, C., Michaelis, H., Hussmann, H., Lingenauber, K., Kallenbach, R., Del Togno, S., Lüdicke, F.: BELA transmitter performance and pointing stability verification campaign at DLRPF. Acta Astronaut. 154, 103-118 (2019)

2. Anderson, J.D., Lau, E.L., Sjogren, W.L., Schubert, G., Moore, W.B.: Gravitational constraints on the internal structure of Ganymede. Nature 384, 541-543 (1996)

3. Araki, H., Tazawa, S., Noda, H., Ishihara, Y., Goossens, S., Sasaki, S., Kawano, N., Kamiya, I., Otake, H., Oberst, J., Shum, C.: Lunar global shape and polar topography derived from Kaguya-LALT Laser altimetry. Science 323, 897-900 (2009)

4. Bagenal, F., Dowling, T.E., McKinnon, W.B. (Eds.): Jupiter, pp. 748. ISBN 0521818087. Cambridge University Press, Cambridge (2004)

5. Boutonnet, A., Varga, G.: JUICE-Jupiter icy moons explorer consolidated report on mission analysis (CReMA), Version 3.1, Technical Note, ESA (2016)

6. Daly, M.G., et al.: The OSIRIS-REx laser altimeter (OLA) investigation and instrument. Space Sci. Rev. 212, 899-924 (2017)

7. Giese, B., Oberst, J., Roatsch, T., Neukum, G.: DLR, institute of planetary exploration J. W. Head and R. T. Pappalardo: the local topography of Uruk Sulcus and Galileo Regio Obtained from stereo images. Icarus 135, 303-316 (1998)

8. Grasset, O., et al.: JUpiter ICy moons Explorer (JUICE): an ESA mission to orbit Ganymede and to characterise the Jupiter system. Planet. Space Sci. 78, 1-21 (2013)

9. Hussmann, H., Shoji, D., Steinbrügge, G., Stark, A., Sohl, F.: Constraints on dissipation in the deep interiors of ganymede and Europa from tidal phase-lags. Cel. Mech. Dyn. Astr. 126, 131-144 (2016)

10. Khurana, K.K., Kivelson, M.G., Stevenson, D.J., Schubert, G., Russel, C.T., Walker, R.J., Polanskey, C.: Induced magnetic fields as evidence for subsurface oceans in Europa and Callisto. Nature 395, 777-780 (1998)

11. Kimura, J., Hussmann, H., Kamata, S., Matsumoto, K., Oberst, J., Steinbrügge, G., Stark, A., Gwinner, K., Oshigami, S., Namiki, N., Lingenauber, K., Enya, K., Kuramoto, K., Sasaki, S.: Science objectives of the ganymede laser altimeter (GALA) for the JUICE mission. Trans. JSASS Aerosp. Tech. Japan 17, 234-243 (2019)

12. Kivelson, M.G., Khurana, K.K., Volwerk, M.: The permanent and inductive magnetic moments on Ganymede. Icarus 157, 507-522 (2002)

13. Li, C., et al.: Laser altimetry data of Chang' $\mathrm{E}-1$ and the global lunar DEM model. Sci. China Earth Sci. 53, 1582-1593 (2010)

14. Moore, W.B., Schubert, G.: The tidal response of Ganymede and Callisto with and without liquid water oceans. Icarus $\mathbf{1 6 6}$, 223-226 (2003)

15. Mukai, T., et al.: An overview of the LIDAR observations of asteroid 25143 Itokawa. Adv. Space Res. 40, 187-192 (2007)

16. Anderson, J.J.D., Schubert, G., Moore, W.B.: Mass anomalies on ganymede: palguta. Icarus 180, 428-441 (2006)

17. Pappalardo, R. T., Collins, G.C., Head III, J. W., Helfenstein, P., McCord, T.B., Moore, J.M., Prockter, L.M., Schenk, P.M., Spencer, J.R.: Geology of Ganymede. In: Jupiter: The planet, satellites and magnetosphere, Bagenal, F., Dowling, T. E. and McKinnon, W. B., Eds., Cambridge University Press, pp. 363-396 (2004)

18. Schubert, G., Anderson, J.D., Spohn, T., McKinnon, W.B.: Interior composition, structure and dynamics of the Galilean satellites. In: Jupiter: The planet, satellites and magnetosphere, Bagenal, F., Dowling, T.E., McKinnon, W.B., Eds., Cambridge University Press, pp. 281-306 (2004)

19. Singer, K.N., Bland, M.T., Schenk, P.M., McKinnon, W.B.: Relaxed impact craters on Ganymede: Regional variation and high heat flows. Icarus 306, 214-224 (2018)

20. Smith, D.E., et al.: Mars Orbiter Laser Altimeter: experiment summary after the first year of global mapping of Mars. J. Geophys. Res. 106(E10), 23689-23722 (2001)

21. Smith, D.E., et al.: The lunar orbiter laser altimeter investigation on the lunar reconnaissance orbiter mission. Space Sci. Rev. 150, 209-241 (2010a)

22. Smith, D.E., et al.: The equatorial shape and gravity field of Mercury from MESSENGER flybys 1 and 2. Icarus 209, 88-100 (2010b)

23. Smith, D.E., et al.: Summary of the results from the lunar orbiter laser altimeter after seven years in lunar orbit. Icarus $\mathbf{2 8 3}, \mathbf{7 0 - 9 1}$ (2017)

24. Spohn, T., Schubert, G.: Oceans in the icy Galilean satellites of Jupiter? Icarus 161, 456-467 (2003)

25. Sohl, F., Spohn, T., Breuer, D., Nagel, K.: Implications from galileo observations on the interior structure and chemistry of the galilean satellites. Icarus 157, 104-119 (2002)

26. Steinbrügge, G., Stark, A., Hussmann, H., Sohl, F., Oberst, J.: Measuring tidal deformations by laser altimetry. A performance model for the Ganymede Laser Altimeter. Planet. Space Sci. 117, 184-191 (2015)

27. Steinbrügge, G., Steinke, T., Thor, R., Stark, A., Hussmann, H.: Measuring ganymede's librations with laser altimetry. Geosciences 9, 320 (2019)

28. Susorney, H.C.M., Barnouin, O.S., Ernst, C.M., Byrne, P.K.: The surface roughness of mercury from the mercury laser altimeter: investigating the effects of volcanism, tectonism, and impact cratering. J. Geophys. Res. (Planets) 22, 1372-1390 (2017)

29. Thomas, N., et al.: The BepiColombo Laser Altimeter (BELA): concept and baseline design. Planet. Space Sci. 55, 1398-1413 (2007)

30. Turcotte, D.L., Schubert, G.: Geodynamics. Cambridge University Press, Cambridge (2002)

31. Zuber, M.T., Smith, D.E., Lemoine, F.G., Neumann, G.A.: The shape and internal structure of the moon from the clementine mission. Science 266, 1839-1843 (1994)

32. Zuber, M.T., et al.: Internal structure and early thermal evolution of mars from mars global surveyor topography and gravity. Science 287, 1788-1793 (2000a)

33. Zuber, M.T., et al.: The shape of 433 Eros from the NEAR-shoemaker laser rangefinder. Science 289, 2097-2101 (2000b)

34. Zuber, M.T., et al.: Topography of the Northern hemisphere of Mercury from MESSENGER laser altimetry. Science 336, $217-$ $220(2012)$

Publisher's Note Springer Nature remains neutral with regard to jurisdictional claims in published maps and institutional affiliations.
805

806

807

808

809

810

811

812

813

814

815

816

817

818

819

820

821

822

823

824

825

826

827

828

829

830

831

832

833

834

835

836

837

838

839

840

841

842

843

844

845

846

847

848

849

850

851

852

853

854

855

856

857 858

859 860 
Journal: $\quad \mathbf{1 2 5 6 7}$

Article: $\quad 282$

\section{Author Query Form}

Please ensure you fill out your response to the queries raised below and return this form along with your corrections

Dear Author

During the process of typesetting your article, the following queries have arisen. Please check your typeset proof carefully against the queries listed below and mark the necessary changes either directly on the proof/online grid or in the 'Author's response' area provided below

\begin{tabular}{|l|l|l|}
\hline Query & Details Required & Author's Response \\
\hline AQ1 & Please check and confirm the processed article title was correct or not. & \\
\hline
\end{tabular}

